Pacific Journal of Mathematics

A CONSTRUCTIVE STUDY OF MEASURE THEORY 


\title{
A CONSTRUCTIVE STUDY OF MEASURE THEORY
}

\author{
YUEN-KWOK CHAN
}

In this paper we study measures on locally compact metric spaces. The constructive theory of a nonnegative measure has been treated in Bishop's book "Foundations of Constructive Analysis". Unfortunately, there is no constructive method to decompose a general signed measure into a difference of two nonnegative ones. In analogy to the classical development, we shall consider two ways to look at a signed measure, namely, as a function function (an integral) and as a set function (a set measure). From an integral on a locally compact metric space $X$ we obtain compact subsets of $X$ to which measures can be assigned. The set measure thus arrived at is shown to be in a weak sense additive, continuous, and of bounded variation. Next we study a set measure having these three properties defined on a large class of compact subsets of $X$. From such a set measure we derive a linear function on the space of test functions of $X$. This linear function is then shown to be an integral. Finally it is demonstrated that the set measure arising from an integral gives rise in this manner to an integral which is equal to the original one. In particular, every integral is the integral arising from some measure (Riesz Representation Theorem).

We shall make use of concepts and results in Bishop's book (reformed to hereafter as C.A.), in which one can find a presentation of the constructive viewpoint and the constructive methods.

1. Compact subsets of a boundedly compact metric space. A metric space $(X, d)$ is said to be totally bounded if, given any $\varepsilon>0$, there is a finite, possibly empty, sequence of points in $X$ which forms an $\varepsilon$-net for $X$. A metric space $(X, d)$ is said to be compact if it is totally bounded and complete. A boundedly compact space ${ }^{1}$ is one in which every bounded subset is contained in some compact subset. Hereafter let $(X, d)$ denote such a space. For each compact subset $A$ of $X$, and each $x \in X$, we let $d(x, A)$ stand for the number min $(1$, inf $\{d(x, y): y \in A\})$. Here the infimum is easily proved to exist (C.A.) if $A$ is nonempty, and is taken to be $\infty$ if $A$ is empty. Given any compact subsets $A$ and $B$ of $X$, write

$$
d^{\prime}(A, B) \equiv \max (0, \sup \{d(x, B): x \in A\}, \sup \{d(x, A): x, B\}) .
$$

Here the supremum of an empty set is taken to be $-\infty . d^{\prime}$ can easily be shown to be a metric on the family of compact subsets of $X$.

1 Called locally compact space in C. A. 
The proof of the following theorem is almost a verbatim reproduction of one in C.A., and so will not be given here.

THEOREM 1.1. Assume $(X, d)$ is compact. Let $f$ be a continuous function on $(X, d)$. Then we can find a countable subset $A=A_{f}$ of the real line, with the following properties.

(1) The set $(a \leqq f \leqq b) \equiv\{x \in X: a \leqq f(x) \leqq b\}$ is compact whenever the numbers $a$ and $b$ are in $-A$ with $a<b$.

(2) Suppose $a$ and $b$ are in $-A$ with $a<b$. Then for numbers $a^{\prime}$ and $b^{\prime}$ in $-A$ which are close enough to $a$ and $b$ respectively, the distance $d^{\prime}\left((a \leqq f \leqq b),\left(a^{\prime} \leqq f \leqq b^{\prime}\right)\right)$ is arbitrarily small.

Definition 1.2. Let $A$ and $B$ be subsets of $X$. We call the closure of $A \cap B$ (resp. $A \cup B$ ) in $X$ the closed intersection (resp. union) of $A$ and $B$, and denote it by $A \wedge B$ (resp. $A \vee B$ ).

Let $K$ and $L$ be compact subsets of $X$. We say $K$ and $L$ are compatible if $K \wedge L$ is compact, and if for each $\varepsilon>0$, there exists $\varepsilon>0$ such that

$$
d(x, K \wedge L)<\varepsilon \text { for every } x \in X \text { with } d(x, K)<\delta \text { and } d(x, L)<\delta \text {. }
$$

We say $K$ well contains $L$, and write $K \supset L$, if there is a positive constant $a$ such that $d(x, L)>a$ whenever $d(x, K)>0$.

Proposition 1.3. Assume that $K, L$, and $H$ are compact subsets and that the sets generated from them by the operations $\wedge$ and $\vee$ are compact and pairwise compatible. Then the following equalities hold.

(i) $K \vee L=L \vee K, K \wedge L=L \wedge K$.

(ii) $(K \vee L) \vee H=K \vee(L \vee H),(K \wedge L) \wedge H=K \wedge(L \wedge H)$.

(iii) $(K \vee L) \wedge H=(K \wedge H) \vee(L \wedge H),(K \wedge L) \vee H=(K \vee H) \wedge$ $(L \vee H)$.

The proof is straightforward and is omitted.

DEFINITION 1.4. For any pair of continuous functions $f$ and $g$ on $X$ we shall let $f \wedge g$ (resp. $f \vee g$ ) denote the function $\min (f, g)$ (resp. $\max (f, g))$.

Suppose $f_{1}, \cdots, f_{n}$ are continuous functions on $X$. We shall let $\Psi=\Psi\left(f_{1}, \cdots, f_{n}\right)$ denote the (finite) family of functions generated from $f_{1}, \cdots, f_{n}$ by the operations $\wedge$ and $\vee$. We shall say a (Lebesgue) null subset $A$ of $R$ is exceptional for the functions $f_{1}, \cdots, f_{n}$ if the following three conditions are satisfied.

(i) For each $a \in-A$, the sets

$$
(f \geqq a) \equiv\{x \in X: f(x) \geqq a\} \quad(f \in \Psi)
$$


are compact.

(ii) For each $a \in-A$ and $f \in \Psi$, by choosing the number $b \in-A$ near enough to $a$, we can make $d^{\prime}((f \geqq a),(f \geqq b))$ arbitrarily small.

(iii) For each $a \in-A$, the $\operatorname{sets}(f \geqq a)(f \in \Psi)$ are pairwise compatible.

The following proposition and its corollary show the abundance of compatible compact sets.

Proposition 1.5. Assume $(X, d)$ is compact. Given continuous functions $f_{1}, \cdots, f_{n}$, there exists an exceptional set $A$ of real numbers for $f_{1}, \cdots, f_{n}$.

Proof. Since $\Psi=\Psi\left(f_{1}, \cdots, f_{n}\right)$ is a finite family, using 1.1 we can find a countable subset $A$ of $R$ such that conditions 1.4 (i)-(ii) hold. Now let $\omega$ be a common modulus of continuity for the functions in $\Psi$. Consider any $a \in-A$, and functions $f$ and $g$ in $\Psi$. Let $\varepsilon>0$ be arbitrary. By 1.4 (ii) we can choose $b \in-A$ with $b<a$ such that

$$
d^{\prime}((f \wedge g \geqq a),(f \wedge g \geqq b))<\varepsilon .
$$

Let $\delta=\min (1, \omega(a-b))$. Suppose $x \in X$ is such that $d(x,(f \geqq a))<\delta$ and $d(x,(g \geqq a))<\delta$. Then there is some $y$ in $(f \geqq a)$ with $d(x, y)<\delta$, and so $f(x) \geqq b$. Similarly $g(x) \geqq b$. Consequently $x \in(f \wedge g \geqq b)$ and so $d(x,(f \wedge g \geqq a))<\varepsilon$. But $(f \wedge g \geqq a)=(f \geqq a) \wedge(g \geqq a)$. It follows that

$$
d(x,(f \geqq a) \wedge(g \geqq a))<\varepsilon .
$$

Therefore $(f \geqq a)$ and $(g \geqq a)$ are compatible. We have thus verified 1.4 (iii).

Definition 1.6. A continuous function $h$ on $(X, d)$ is said to be proper if for all real numbers $a$ and $b$ with $a<b$, the set $(a \leqq h \leqq b)$ is contained in some compact subset of $X$.

CoROLLARY 1.7. Let $f_{1}, \cdots f_{n}$ be proper functions on $X$ which are bounded above. Then there is a null subset $A$ of $R$ which is exceptional for $f_{1}, \cdots, f_{n}$.

Proof. For each natural number $k$, choose a real number $a_{k}$ such that $a_{k}<-k$, and such that the set $L_{k} \equiv\left(f_{1} \vee \cdots \vee f_{n} \geqq a_{k}\right)$ is compact. Choose a compact set $K_{k}$ which well contains $L_{k}$. Then by 1.5 there is a null set $A_{k}$ of real numbers which is exceptional for the functions $f_{1}\left|K_{k}, \cdots, f_{n}\right| K_{k}$. Since compact sets in $L_{k}$ which are compatible as compact subsets of $K_{k}$ are also compatible in $X$, the set of real numbers $\bigcup_{k=1}^{\infty} A_{k}$ can easily be verified to be exceptional for the functions $f_{1}, \cdots f_{n}$. 
Proposition 1.8. Suppose the null set $A$ is exceptional for the functions $f_{1}, \cdots, f_{n}$. Then for all $f$ and $g$ in $\Psi\left(f_{1}, \cdots, f_{n}\right)$ and $a \in-A$, we have $(f \wedge g \geqq a)=(f \geqq a) \wedge(g \geqq a)$ and $(f \vee g \geqq a)=(f \geqq a) \vee(g \geqq a)$.

Proof. The first equality is easily verified. Take any $x$ in $(f \vee g \geqq$ $a)$. For every natural number $k$ choose $b_{k}$ in $-A$ with $b_{k}<a$ and $d^{\prime}\left(\left(f \geqq b_{k}\right),(f \geqq a)\right)<k^{-1}$. Then either $x \in(f<a)$ or $x \in\left(f \geqq b_{k}\right)$. In either case $d(x,(f \geqq a) \vee(g \geqq a))$ is less than $k^{-1}$. Thus $x \in(f \geqq a) \vee$ $(g \geqq a)$. So $(f \vee g \geqq a)$ is contained in $(f \geqq a) \vee(g \geqq a)$. Containment in the other direction is obvious.

2. Measure induced by an integral. Hereafter $C(X)$ will denote the space of test functions on $(X, d)$, namely, continuous functions on $X$ with compact supports.

DEFINITION 2.1. A (signed) integral $\mu$ on $X$ is a linear function on $C(X)$ whose value at an element $f$ of $C(X)$ is written $\int f d u$, such that for each $f \in C(X)$ there is $M_{f} \geqq 0$ with $\left|\int h d \mu\right| \leqq M_{f}$ whenever $h \in C(X)$ and $|h| \leqq|f|$.

DEFINITION 2.2. A sequence $\left\{f_{n}\right\}$ of test functions is said to belong to a compact subset $K$ of $X$ if for all $n$,

(i) $0 \leqq f_{n} \leqq 1$,

(ii) $f_{n}(x)=0$ if $d(x, K) \geqq n^{-1}$, and (iii) $f_{n}(x)=1$ if $x \in K$.

In the following, let $\mu$ be a given integral on $X$.

Definition 2.3. A compact subset $K$ of $X$ is said to be strongly measurable with respect to $\mu$ if there is a sequence $\left\{f_{n}\right\}$ of test functions belonging to $K$ such that $\int f_{n} g d \mu$ converges for all test functions $g$.

The following lemma is proved in C.A. (P. 173).

Lemma 2.4. Let $K$ be a compact subset of $X$. Suppose $\left\{f_{n}\right\}$ belongs to $K$ and suppose $\int f_{n} d \mu$ converges. Then the limit is independent of the sequence $\left\{f_{n}\right\}$. Thus if $\left\{f_{n}\right\}$ is in addition such that $\int f_{n} g d \mu$ converges for all $g \in C(X)$, then, for given $g$, the limit $\lim _{n \rightarrow \infty} \int f_{n} g d \mu$ is independent of $\left\{f_{n}\right\}$.

Definition 2.5. Let $K$ be strongly measurable and let $g \in C(X)$. Define $\mu(K) \equiv \lim _{n \rightarrow \infty} \int f_{n} d \mu$ and $\int_{K} g d \mu \equiv \lim _{n \rightarrow \infty} \int f_{n} g d \mu$, where $\left\{f_{n}\right\}$ is some 
sequence of test functions belonging to $K$ such that these limits exist. (By 2.4 these are well defined). $\mu(K)$ is called the strong measure of K.

Proposition 2.6. Let $K$ be strongly measurable and $g$ a test function. From every sequence $\left\{f_{n}^{\prime}\right\}$ of test functions belonging to $K$, we can select a subsequence $\left\{f_{n}^{\prime \prime}\right\}$ such that

$$
\lim _{n \rightarrow \infty} \int f_{n}^{\prime \prime} g d \mu=\int_{K} g d \mu \text {. }
$$

Proof. Let $\left\{f_{n}\right\}$ be a sequence of test functions belonging to $K$ such that $\int f_{n} h d \mu$ converges for all $h \in C(X)$. Since $\left|f_{n}-f_{n}^{\prime}\right|$ vanishes on $K$ and on $\left\{x \in X: d(x, K) \geqq n^{-1}\right\}$, we can select a sequence of integers $\left\{n_{i}\right\}$ such that $n_{i}<n_{i+1}$ and

$$
\left|f_{n_{1}}-f_{n_{1}}^{\prime}\right|+\cdots+\left|f_{n_{i}}-f_{n_{i}}^{\prime}\right| \leqq 2 \text { for all } i \text {. }
$$

By abuse of notation we again write $f_{i}$ for $f_{n_{i}}$, and $f_{i}^{\prime}$ for $f_{n_{i}}^{\prime}$. Let $h_{i}=f_{i}-f_{i}^{\prime}(i=1,2, \cdots)$. Then clearly for every choice of integers $j$ and $i_{1}<i_{2}<\cdots<i_{j}$ we have

$$
\left|h_{i_{1}}+\cdots+h_{i_{j}}\right| \leqq 2 \quad \text { on } X \text {. }
$$

Therefore

$$
\left|h_{i_{1}} g+\cdots+h_{i_{j}} g\right| \leqq 2|g| \quad \text { on } X,
$$

and so, by the definition of an integral, there is $M \geqq 0$ such that

$$
\left|\int h_{i_{1}} g d \mu+\cdots+\int h_{i_{j}} g d \mu\right| \leqq M
$$

for all integers $j$ and $i_{1}<i_{2}<\cdots<i_{j}$. Thus, by passing to a subsequence, we may assume

$$
\int h_{i} g d \mu \rightarrow 0 \quad \text { as } i \rightarrow \infty \text {. }
$$

If follows that $\int f_{i}^{\prime} g d \mu \rightarrow \lim _{n \rightarrow \infty} \int f_{n} g d \mu$.

Thus $\int_{n}^{\prime} f_{n}^{\prime} g d \mu$ converges in a weak sense to $\int_{K} g d \mu$ (Given any subsequence of $\int f_{n}^{\prime} g d \mu$ we can select a subsequence which converges to $\left.\int_{K} g d \mu\right)$. This is the strongest result we can expect to get, as is shown by the following example.

Let $\left\{a_{n}\right\}$ be a given sequence of 0 or 1 's, containing at most one 1 , but it is not known whether there is a 1 . For each $n$ write $r_{n}$ for $n^{-1}$. Define integrals $\mu_{n}$ on the real line by $\mu_{n}=\varepsilon_{2 n}-\varepsilon_{2 n+1}$ where $\varepsilon_{k}$ 
denotes the integral defined by $\int f d \varepsilon_{k}=f\left(r_{k}\right)$ for all $f \in C(R)$. Let $\mu$ be the integral on $R$ defined by

$$
\int f d \mu=\sum_{n=1}^{\infty} a_{n} \int f d \mu_{n} \quad \text { for all } f \in C(R) .
$$

The last series converges by the continuity of $f$. For each $n$, let $f_{n}$ be a continuous function on $R$, having values in [0,1], supported by $\left[-r_{2 n-1}, r_{2 n-1}\right]$, and equal to 1 on $\left[-r_{2 n}, r_{2 n}\right]$. Let $f_{n}^{\prime}$ be a continuous function on $R$, having values in $[0,1]$, supported by $\left[-r_{2 n}, r_{2 n}\right]$, and equal to 1 on $\left[-r_{2 n+1}, r_{2 n+1}\right]$. Clearly $f_{n}$ and $f_{n}^{\prime}$ belong to the compact set $K=\{0\}$. For each $n$ and $m$ it is also clear that $\int f_{n} d \mu_{m}=0$. Thus $\int f_{n} d \mu=0$ for all $n$. Given any test function $g$, since $g$ behaves like a constant near 0 , we can prove $\int f_{n} g d \mu \rightarrow 0$. Thus $K$ is strongly measurable with respect to $\mu$. On the other hand for any $n$ and $m$ we have $\int f_{n}^{\prime} d \mu_{m}=-\delta_{m n}$ ( $\delta_{m n}$ the Kronecker delta). Hence $\int f_{n}^{\prime} d \mu=$ $-a_{n}$. We cannot, however, tell whether $-a_{n}$ converges in the ordinary sense.

LEMMA 2.7. If $K$ is strongly measurable, and $g$ and $g^{\prime}$ are test functions which coincide on $K$, then $\int_{K} g d \mu=\int_{K} g^{\prime} d \mu$.

Proof. Let $\left\{f_{n}\right\}$ be a sequence of test functions belonging to $K$ such that $\int f_{n} h d \mu$ converges for all $h \in C(X)$.

There exists a compact set $L$ outside which $g$ and $g^{\prime}$ vanish. Let $f$ be a test function which equals 1 on $L$. Then by the definition of an integral, there is $M_{f} \geqq 0$ such that

$$
\left|\int h d \mu\right| \leqq M_{f}\|h\|
$$

wherever $h \in C(X)$ is supported by $L$. Now $g-g^{\prime}$ vanishes on $K$ and so $\left\|\left(g-g^{\prime}\right) f_{n}\right\|$ is arbitrarily small if $n$ is large enough. But

$$
\left|\int\left(g-g^{\prime}\right) f_{n} d \mu\right| \leqq M_{f}\left\|\left(g-g^{\prime}\right) f_{n}\right\| \text {. }
$$

The desired result follows.

Definition 2.8. Let $K$ be strongly measurable. For each continous function $\bar{g}$ on $K$, write

$$
\mu_{K}(\bar{g}) \equiv \int \bar{g} d \mu_{K} \equiv \int_{K} g d \mu
$$


where $g$ is some test function on $X$ which extends $\bar{g} . \quad \mu_{K}$ is a function by 2.7. Clearly $\mu_{K}$ is an integral. We call $\mu_{K}$ the restriction of $\mu$ to $K$.

Proposition 2.9. Suppose $K$ is a compact subset of $X$ which is strongly measurable with respect to $\mu$. Suppose $L$ is a compact subset of $X$ which is well containd in $K$ and strongly measurable with respect to $\mu_{K}$. Then $L$ is strongly measurable with respect to $\mu$, and $\mu(L)=\mu_{K}(L)$.

Proof. Let $\left\{f_{n}\right\}$ be a sequence of test functions on $X$ belonging to $K$, such that $\int f_{n} g d \mu$ converges for all $g \in C(X)$. Let $\left\{f_{n}^{\prime}\right\}$ be a sequence of test functions on $K$ bearing a similar relationship to $L$ and $\mu_{K}$. Since $K \supset \supset L$, for each $n$ we may assume that $f_{n}^{\prime}$ is the restriction to $K$ of some test function $f_{n}^{\prime \prime}$ on $X$ which is supported by $K$; indeed we may assume that the sequence $\left\{f_{n}^{\prime \prime}\right\}$ belongs to $L$. Now, for any $g \in C(X)$, let $g_{K}$ be the restriction of $g$ to $K$. Then by assumption the sequence $\int f_{n}^{\prime} g_{K} d \mu_{K}$ converges. But, for each $n$,

$$
\int f_{n}^{\prime} g_{K} d \mu_{K}=\lim _{m \rightarrow \infty} \int f_{m} f_{n}^{\prime \prime} g d \mu=\int f_{n}^{\prime \prime} g d \mu .
$$

The first equality holds by the definition of $\mu_{K}$; the second because $f_{n}^{\prime \prime} g$ is supported by $K$, and $f_{m}=1$ on $K$. Thus $\int f_{n}^{\prime \prime} g d \mu$ converges. Moreover

$$
\mu(L)=\lim _{n \rightarrow \infty} \int f_{n}^{\prime \prime} d \mu=\lim _{n \rightarrow \infty} \int f_{n}^{\prime} d \mu_{K}=\mu_{K}(L) .
$$

The following theorem, which is a generalization of one in C.A., shows that strongly measurable sets are abundant.

THEOREM 2.10. Let $h$ be a proper function on $X$. Then there exists a (Lebesgue) null set $B$ of real numbers such that the set $(u \leqq h \leqq v)$ is strongly measurable for all $u$ and $v$ in $-B$ with $u<v$.

Proof. In view of 2.9, we may assume that $X$ is compact. It suffices to show that for $[0,1]$ (and similarly for any interval) there is a null set $B$ such that the set $(u \leqq h \leqq v)$ is strongly measurable for all $u$ and $v$ in $[0,1]-B$ with $u<v$. Without loss of generality, assume that $\left|\int f d \mu\right| \leqq\|f\|$ for every $f \in C(X)$.

For each natural number $n$ write

$$
G_{n} \equiv\{g \in C(X):\|g\| \leqq 1 \text { and }|g(x)-g(y)| \leqq n d(x, y)\},
$$


and write for each $k\left(0 \leqq k \leqq n^{4}-1\right)$,

$$
\begin{aligned}
& S_{n k} \equiv\{f \in C(R):\|f\| \leqq 1 ; \quad f \text { is supported by } \\
& \left.\left[k n^{-4},(k+1) n^{-4}\right] ; \text { and }|f(s)-f(t)| \leqq 2(n+1)^{6}|s-t|\right\} .
\end{aligned}
$$

Then, by Ascoli's Theorem (C.A.), the sets $G_{n}$ and $S_{n k}$ are compact with respect to the supremum norms. Therefore, for given natural numbers $m, n$, and $k\left(0 \leqq k \leqq n^{4}-1\right)$, the number

$$
a_{m n k} \equiv \sup \left\{\int(f \circ h) g d \mu: f \in S_{n k}, g \in G_{m}\right\}
$$

is well defined. For given $m$ and $n$, let $U_{m n}$ and $V_{m n}$ be a partition of $\left\{0,1, \cdots, n^{4}-1\right\}$ such that

$$
a_{m n k}>n^{-2} \quad \text { if } \quad k \in U_{m n} ; \quad a_{m n k}<2 n^{-2} \quad \text { if } k \in V_{m n} \text {. }
$$

For each $k \in U_{m n}$, choose $f_{k} \in S_{n k}$ and $g_{k} \in G_{m}$ with

$$
\int\left(f_{k} \circ h\right) g_{k} d \mu>n^{-2} \text {. }
$$

Let $f=\sum_{k \in U_{m n}}\left(f_{k} \circ h\right) g_{k}$ Then $\|f\| \leqq 1$ by the definition of the $f_{k}^{\prime}$ s. Thus

$$
1 \geqq \int f d \mu=\sum_{k \in U_{m n}} \int\left(f_{k} \circ h\right) g_{k} d \mu>n^{-2} \text { card }\left(U_{m n}\right) .
$$

Or card $\left(U_{m n}\right) \leqq n^{2} . \quad\left(\operatorname{card}\left(U_{m n}\right)\right.$ is the number of elements in $\left.U_{m n}\right)$.

Now construct a countable subset $A_{h}$ of $R$ which is related to $h$ as $A_{f}$ is to $f$ in 1.1. For all natural numbers $m$ and $n$, let $B_{m n}$ be the union of the set

$$
\bigcup_{k \in U_{m n}}\left[k n^{-4},(k+1) n^{-4}\right]
$$

and the set

$$
\bigcup_{0 \leqq k \leqq n^{4}}\left(\left[k n^{-4}-n^{-6}, k n^{-4}+n^{-6}\right] \cap[0,1]\right) .
$$

Then the Lebesgue measure of $B_{m n}$ is at most

$$
n^{-4} \text { card }\left(U_{m n}\right)+2 n^{4} \cdot n^{-6} \leqq 2 n^{-2} .
$$

Thus $B \equiv A_{h} \cup\left(\bigcup_{m=1}^{\infty} \bigcap_{j=1}^{\infty} \bigcup_{m=j}^{\infty} B_{m n}\right)$ has Lebesgue measure zero. Now suppose $u, v \in[0,1]-B$ with $u<v$. We shall show that the set $(u \leqq h \leqq v)$ is strongly measurable.

For each $n$, construct $f_{n} \in C(R)$ with support $\left[u-n^{-6}, v+n^{-6}\right]$ such that $f_{n}=1$ on $[u, v]$ and $f_{n}$ is linear on each of the intervals $\left[u-n^{-6}, u\right]$ and $\left[v, v+n^{-6}\right]$. Let $m$ be an arbitrary natural number, and $g \in G_{m}$. There is a natural number $j$ such that $u, v \in-B_{m n}$ for 
all $n \geqq j . \quad$ Fix any $n$ with $n \geqq j$. It follows from the definition of $B_{m n}$ that

$$
\begin{aligned}
& u \in\left[k^{\prime} n^{-4}+n^{-6},\left(k^{\prime}+1\right) n^{-4}-n^{-6}\right] \text { for some } k^{\prime} \in V_{m n} ; \\
& v \in\left[k^{\prime \prime} n^{-4}+n^{-6},\left(k^{\prime \prime}+1\right) n^{-4}-n^{-6}\right] \text { for some } k^{\prime \prime} \in V_{m n}\left(k^{\prime} \leqq k^{\prime \prime}\right) .
\end{aligned}
$$

Since $f_{n}-f_{n+1}$ is supported by $\left[u-n^{-6}, u\right] \cup\left[v, v+n^{-6}\right]$ and since $u<v$, we can write $f_{n}-f_{n+1}=f^{\prime}+f^{\prime \prime}$ where $f^{\prime}$ equals $f_{n}-f_{n+1}$ on $\left[u-n^{-6}, u\right]$ and zero elsewhere, and where $f^{\prime \prime}$ equals $f_{n}-f_{n+1}$ on $\left[v, v+n^{-6}\right]$ and zero elsewhere. In particular, $f^{\prime}$ is supported by $\left[k^{\prime} n^{-4},\left(k^{\prime}+1\right) n^{-4}\right]$. For all real numbers $s$ and $t$,

$$
\begin{aligned}
\left|f^{\prime}(s)-f^{\prime}(t)\right| & \leqq\left|f_{n}(s)-f_{n}(t)\right|+\left|f_{n+1}(s)-f_{n+1}(t)\right| \\
& \leqq 2(n+1)^{6}|s-t|
\end{aligned}
$$

Therefore $f^{\prime} \in S_{n k^{\prime}}$. But $k^{\prime} \in V_{m n}$. Thus $a_{m n k^{\prime}}<2^{n-2}$. Consequently, $\left|\int\left(f^{\prime} \circ h\right) g d \mu\right| \leqq 2 n^{-2}$. Similarly we show $\left|\int\left(f^{\prime \prime} \circ h\right) g d \mu\right| \leqq 2 n^{-2}$. Combining, we see that

$$
\begin{aligned}
& \left|\int\left(f_{n} \circ h\right) g d \mu-\int\left(f_{n+1} \circ h\right) g d \mu\right| \\
& \quad \leqq\left|\int\left(f^{\prime} \circ h\right) g d \mu\right|+\left|\int\left(f^{\prime \prime} \circ h\right) g d \mu\right| \leqq 4 n^{-2} \quad(n \geqq j) .
\end{aligned}
$$

Therefore $\int\left(f_{n} \circ h\right) g d \mu$ converges for all $g \in G_{m}$. Since by the StoneWeierstrass Theorem (C.A.), the linear span of $\bigcup_{m=1}^{\infty} G_{m}$ is dense in $C(X)$ under the supremum norm, we see that $\int\left(f_{n} \circ h\right) g d \mu$ convergers for all $g \in C(X)$. It remains to choose from the sequence $\left\{f_{n} \circ h\right\}$ a subsequence which belongs to the set $(u \leqq h \leqq v)$. For every natural number $i$, by the definition of $B$ and $A_{h}$, we can find $u^{\prime}$ and $v^{\prime}$ in $[0,1]-B$ such that $u^{\prime}<u<v<v^{\prime}$ and

$$
d^{\prime}\left((u \leqq h \leqq v),\left(u^{\prime} \leqq h \leqq v^{\prime}\right)\right) \leqq i^{-1} .
$$

Let $n_{i}$ be a natural number greater than $\left(u-u^{\prime}\right)^{-1}$ and $\left(v^{\prime}-v\right)^{-1}$. For every $x$ such that $d(x,(u \leqq h \leqq v))>i^{-1}$, we have $h(x) \leqq u^{\prime}$ or $h(x) \geqq v^{\prime}$. It follows that $f_{n_{i}} \circ h$ vanishes at this $x$. Clearly $f_{n_{i}} \circ h=1$ on the set $(u \leqq h \leqq v)$. Combining, we see that $\left\{f_{n_{i}} \circ h\right\}$ belongs to $(u \leqq h \leqq v)$.

Proposition 2.11. If $\left\{K_{n}\right\}$ is a sequence of strongly measurable subsets of $X$, if $K$ is a strongly measurable subset contained in each $K_{n}$, and if $d^{\prime}\left(K, K_{n}\right) \leqq n^{-1}$ for each $n$, then $\mu\left(K_{n}^{\prime}\right)$ converges to $\mu(K)$ for some subsequence $\left\{K_{n}^{\prime}\right\}$ of $\left\{K_{n}\right\}$. 
Proof. Choose a sequence $\left\{g_{n}\right\}$ of test functions such that $0 \leqq g_{n} \leqq 1$, $g_{n}=1$ on $K_{n}, g_{n}(x)=0$ if $d\left(x, K_{n}\right) \geqq n^{-1}$, and $\left|\int g_{n} d \mu-\mu\left(K_{n}\right)\right| \leqq n^{-1}$. Since $d^{\prime}\left(K, K_{n}\right) \leqq n^{-1}$, the sequence $\left\{g_{2 n}\right\}$ belongs to $K$. Hence by 2.7 we can choose a subsequence $\left\{g_{n}^{\prime}\right\}$ of $\left\{g_{2 n}\right\}$ such that $\int g_{n}^{\prime} d \mu \rightarrow \mu(K)$ as $n \rightarrow \infty$. Take the corresponding subsequence $\left\{K_{n}^{\prime}\right\}$ of $\left\{K_{2 n}\right\}$. Obviously $\mu\left(K_{n}^{\prime}\right) \rightarrow \mu(K)$.

Lemma 2.12. Suppose $K$ is strongly measurable. Then there exists a constant $A_{k} \geqq 0$ such that

$$
\sum_{i=1}^{m}\left|\mu\left(L_{i}\right)-\mu\left(K_{i}\right)\right| \leqq A_{K}
$$

for every sequence $L_{1}, K_{1}, L_{2}, K_{2}, \cdots, L_{m}, K_{m}$ of strongly measurable sets with $K \supset \supset L_{1} \supset \supset K_{1} \supset \supset \cdots \supset \supset L_{m} \supset \supset K_{m}$.

Proof. By the definition of an integral, there exists a constant $A_{K} \geqq 0$ such that $\left|\int f d \mu\right| \leqq A_{K}\|f\|$ for each $f \in C(X)$ supported by $K$. Now let

$$
K \supset \supset L_{1} \supset \supset K_{1} \supset \supset \cdots \supset \supset L_{m} \supset \supset K_{m}
$$

be a sequence of strongly measurable sets. Choose sequences of test functions $\left\{f_{n}^{i}\right\}$ and $\left\{g_{n}^{i}\right\}$ belonging to $K_{i}$ respectively $L_{i}$ such that

$$
\lim _{n \rightarrow \infty} \int f_{n}^{i} d \mu=\mu\left(K_{i}\right) ; \quad \lim _{n \rightarrow \infty} \int g_{n}^{i} d \mu=\mu\left(L_{i}\right) \quad(1 \leqq i \leqq m) .
$$

Let $\delta>0$ be arbitrary. Partition $1, \cdots, m$ into subsets $P$ and $Q$ such that

$$
u\left(L_{i}\right)-\mu\left(K_{i}\right)<\delta \quad \text { if } \quad i \in P ; \mu\left(L_{i}\right)-\mu\left(K_{i}\right)>-\delta \text { if } i \in Q .
$$

Then

$$
\begin{aligned}
& \sum_{i=1}^{m}\left|\left(L_{i}\right)-\mu\left(K_{i}\right)\right|=\sum_{i \in P}\left|\mu\left(L_{i}\right)-\mu\left(K_{i}\right)\right|+\sum_{i \in Q}\left|\mu\left(L_{i}\right)-\mu\left(K_{i}\right)\right| \\
& \quad \leqq \sum_{i \in P}\left\{-\left(\mu\left(L_{i}\right)-\mu\left(K_{i}\right)\right)+2 \delta\right\}+\sum_{i \in Q}\left\{\mu\left(L_{i}\right)-\mu\left(K_{i}\right)+2 \delta\right\} \\
& =2 m \delta+\lim _{n \rightarrow \infty}\left\{\sum_{i \in P} \int\left(f_{n}^{i}-g_{n}^{i}\right) d \mu+\sum_{i \in Q} \int\left(g_{n}^{i}-f_{n}^{i}\right) d \mu\right\} .
\end{aligned}
$$

But, from the well containment $(*)$, it follows that if $n$ is large enough, the function $\sum_{i \in P}\left(f_{n}^{i}-g_{n}^{i}\right)+\sum_{i \in Q}\left(g_{n}^{i}-f_{n}^{i}\right)$ is supported by $K$ and has values in $[-1,1]$. Therefore by the definition of $A_{K}$, the integral of this function 


$$
\sum_{i \in P} \int\left(f_{n}^{i}-g_{n}^{i}\right) d \mu+\sum_{i \in Q} \int\left(g_{n}^{i}-f_{n}^{i}\right) d \mu
$$

is at most $A_{K}$ if $n$ is large enough.

Consequently

$$
\sum_{i=1}^{m}\left|\mu\left(L_{i}\right)-\mu\left(K_{i}\right)\right| \leqq 2 m \delta+A_{K} \cdot
$$

But $\delta$ was arbitrary. The desired inequality follows.

Proposition 2.13. Suppose $K$ is strongly measurable. Then there exists a constant $B_{K}>0$ such that

$$
\sum_{i=1}^{m-1}\left|\mu\left(K_{i}\right)-\mu\left(K_{i+1}\right)\right| \leqq B_{K}
$$

for every sequence of strongly measurable sets $K \supset K_{1} \supset \cdots \supset K_{m}$.

Proof. Choose a positive real number $a_{0}$ such that the set

$$
K^{\prime} \equiv\left\{x \in X: \quad d(x, K) \leqq a_{0}\right\}
$$

is strongly measurable. Such a choice is possible by 2.10 . Let $A_{K^{\prime}} \geqq 0$ be a constant associated to $K^{\prime}$ as in the above lemma. Let $B_{K}=$ $2 A_{K^{\prime}}+1$. Now consider any sequence of strongly measurable sets $K \supset K_{1} \supset K_{2} \supset \cdots \supset K_{m}$. Inductively (on $i=1, \cdots, m$ ), let $a_{i}>0$ be so small that $a_{i}<a_{i-1}$ and such that

$$
K_{i}^{\prime} \equiv\left\{x \in X: \quad d\left(x, K_{i}\right) \leqq a_{i}\right\}
$$

is strongly measurable with $\left|\mu\left(K_{i}\right)-\mu\left(K_{i}^{\prime}\right)\right|<(2 m)^{-1}$. Then it can easily be shown that

$$
K^{\prime} \supset \supset K_{1}^{\prime} \supset \supset K_{2}^{\prime} \supset \supset \cdots \supset \supset K_{m}^{\prime} \text {. }
$$

Therefore, by the definition of $A_{K^{\prime}}$,

$$
\sum_{i=1}^{m-1}\left|\mu\left(K_{i}^{\prime}\right)-\mu\left(K_{i+1}^{\prime}\right)\right| \leqq 2 A_{K^{\prime}} \cdot
$$

But then

$$
\sum_{i=1}^{m-1}\left|\mu\left(K_{i}\right)-\mu\left(K_{i+1}\right)\right| \leqq 2 A_{K^{\prime}}+1=B_{K}
$$

Proposition 2.14. If two strongly measurable sets $K_{1}$ and $K_{2}$ are compatible, and $K_{1} \wedge K_{2}$ as well as $K_{1} \vee K_{2}$ are strongly measurable, then

$$
\mu\left(K_{1}\right)+\mu\left(K_{2}\right)=\mu\left(K_{1} \wedge K_{2}\right)+\mu\left(K_{1} \vee K_{2}\right) .
$$


Proof. Let $\left\{f_{n}\right\},\left\{g_{n}\right\}$ be sequences of test functions belonging to $K_{1}$ and $K_{2}$ respectively. Clearly the sequence $\left\{f_{n} \vee g_{n}\right\}$ belongs to $K_{1} \vee K_{2}$. Since $K_{1}$ and $K_{2}$ are compatible we can select a sequence $n_{1}<n_{2}<\cdots<n_{k}<\cdots$ of natural numbers such that $d\left(x, K_{1} \wedge K_{2}\right)>$ $k^{-1}$ implies $d\left(x, K_{1}\right)>n_{k}^{-1}$ or $d\left(x, K_{2}\right)>n_{k}^{-1}$. Then the sequence $\left\{f_{n_{k}} \wedge g_{n_{k}}\right\}$ belongs to $K_{1} \wedge K_{2}$. Therefore, by 2.6 and by passing to a subsequence, we have

$$
\begin{aligned}
\mu\left(K_{1} \wedge K_{2}\right)+\mu\left(K_{1} \vee K_{2}\right) & =\lim _{k \rightarrow \infty}\left(\int f_{n_{k}} \wedge g_{n_{k}} d \mu+\int f_{n_{k}} \vee g_{n_{k}} d \mu\right) \\
& =\lim _{k \rightarrow \infty} \int\left(f_{n_{k}}+g_{n_{k}}\right) d \mu=\mu\left(K_{1}\right)+\mu\left(K_{2}\right) .
\end{aligned}
$$

Proposition 2.15. The empty set $\varnothing$ is strongly measurable, and $\mu(\varnothing)=0$.

Proof. Just consider the sequence of test functions $\left\{f_{n}\right\}$ where $f_{n} \equiv 0$ for each $n$.

3. Measure spaces and integration.

DEFINITION 3.1. Let $(X, d)$ be a boundedly compact metric space. Let $F$ be a family of compact subsets of $X$ such that, for every proper function $f$ on $X$, there is a (Lebesgue) null subset $A_{f}$ of $R$ such that the set $(a \leqq f \leqq b)$ is in $F$ for all $a$ and $b$ in $-A_{f}$ with $a<b$. Suppose further $\nu$ is a real valued function on $F$ such that

(i) $\nu$ is additive in the sense that

$$
\nu\left(K_{1}\right)+\nu\left(K_{2}\right)=\nu\left(K_{1} \wedge K_{2}\right)+\nu\left(K_{1} \vee K_{2}\right)
$$

whenever $K_{1}, K_{2} \in F$ are compatible and $K_{1} \wedge K_{2}, K_{1} \vee K_{2}$ are in $F$,

(ii) $\nu$ is continuous in the sense that, given compact sets $K, K_{n} \in$ $F(n \in N)$ with $K \subset K_{n}$ and $d^{\prime}\left(K, K_{n}\right) \rightarrow 0$, we can choose a subsequence $\left\{K_{n}^{\prime}\right\}$ of $\left\{K_{n}\right\}$ such that $\nu\left(K_{n}^{\prime}\right) \rightarrow \nu(K)$,

(iii) $\nu$ is of bounded variation in the sense that, for every $K$ in $F$ there exists $B_{K} \geqq 0$ such that for any sequence $K \supset K_{1} \supset K_{2} \supset \cdots \supset K_{m}$ of elements of $F$ we have

$$
\sum_{i=1}^{m-1}\left|\nu\left(K_{i}\right)-\nu\left(K_{i+1}\right)\right| \leqq B_{K},
$$

(iv) $\nu(\varnothing)=0$, where $\varnothing$ is the empty set.

Then we say that $\nu$ is a signed measure on $(X, d)$ and $(X, d, F, \nu)$ is a signed measure space. When no confusion is likely, we call a signed measure simply a measure, and a signed measure space simply a measure space. Members of $F$ are said to be measurable. 
In what follows, $(X, d, F, \nu)$ will denote a given measure space, unless otherwise is explicitly stated.

Lemma 3.2. Let $K$ and $L$ in $F$ be such that $\nu(K) \neq \nu(L)$. Then $d^{\prime}(K, L)>0$.

Proof. Construct a sequence $\left\{a_{n}\right\}$ of 0 or 1's such that

$$
d^{\prime}(K, L)<n^{-1} \quad \text { if } \quad a_{n}=0 ; \quad d^{\prime}(K, L)>0 \quad \text { if } \quad a_{n}=1 .
$$

For each natural number $n$ such that $a_{n}=0$, choose a positive real number $b_{n}$ with $b_{n}<n^{-1}$ such that the set

$$
K_{n} \equiv L_{n} \equiv\left\{x \in X: \quad d(x, K) \leqq b_{n}\right\}
$$

is compact and belongs to $F$. By the definition of $F$ this choice is possible. For each $n$ such that $a_{n}=1$, let $K_{n}=K$ and $L_{n}=L$. Obviously $d^{\prime}\left(K, K_{n}\right) \leqq n^{-1}$ and $d^{\prime}\left(L, L_{n}\right) \leqq 2 n^{-1}$ for all $n$. Therefore by 3.1 (ii) we can find an $n$ such that

$$
\left|\nu\left(K_{n}\right)-\nu(K)\right|<2^{-1}|\nu(L)-\nu(K)|
$$

and

$$
\left|\nu\left(L_{n}\right)-\nu(L)\right|<2^{-1}|\nu(L)-\nu(K)| \cdot
$$

For this $n$, we cannot have $a_{n}=0$, because this would imply $K_{n}=L_{n}$, contradicting the above inequalities. But then $a_{n}=1$ and so $d^{\prime}(K, L)>$ 0 .

In the following let $g$ be a test function and $\alpha>0$ be some real number such that $(|g| \geqq \alpha)$ is compact. Suppose $x_{1}, \cdots, x_{n}$ form a $\beta$ net for $(|g| \geqq \alpha)$ where $\beta$ is some positive real number such shat $\beta<\alpha$ and $K_{i}=\left\{x: d\left(x, x_{1}\right) \wedge \cdots \wedge d\left(x, x_{i}\right) \leqq \beta\right\}$ belong to $F$ for every $i=$ $1, \cdots, n$. If $-\beta$ belongs to the complement of some exceptional set for $-d\left(\cdot, x_{1}\right)(i=1, \cdots, n)$, write

$$
S=\sum_{i=1}^{n} g\left(x_{i}\right)\left(\nu\left(K_{i}\right)-\nu\left(K_{i-1}\right)\right),
$$

where for convenience we write $K_{0}$ for the empty set. We will show that as $\alpha$ approaches 0 , the sum $S$ converges.

Proposition 3.3. With notation as above, the limit $\lim _{\alpha \rightarrow 0} S$ exists.

Proof. Consider two sums $S=\sum_{i=1}^{n} g\left(x_{i}\right)\left(\nu\left(K_{i}\right)-\nu\left(K_{i-1}\right)\right)$ where $x_{1}, \cdots, x_{n}$ form a $\beta$-net for $(|g| \geqq \alpha)$, and $S^{\prime}=\sum_{j=1}^{m}\left(g_{j}^{\prime}\right)\left(\nu\left(K_{j}^{\prime}\right)-\nu\left(K_{j-1}^{\prime}\right)\right)$ where $x_{1}^{\prime}, \cdots, x_{m}^{\prime}$ form a $\beta^{\prime}$-net $f$ or $\left(|g| \geqq \alpha^{\prime}\right)$. In proving that $\left|S-S^{\prime}\right|$ is arbitrarily small if $\alpha$ and $\alpha^{\prime}$ are small enough, we may assume that 
$K_{i}$ and $K_{j}^{\prime}(i=1, \cdots, n ; j=1, \cdots, m)$ are such that the compact sets obtained from them via the operations $\wedge$ and $\vee$ belong to $F$ and are pairwise compatible. (If necessary we can replace $\beta$ and $\beta^{\prime}$ by $\beta+\theta$ and $\beta^{\prime}+\theta$ respectively, where $\theta$ is chosen according to the following restrictions. Firstly $-\theta$ belongs to the complement of some exceptional set for the functions generated via $\wedge$ and $\vee$ from the functions $\beta-$ $d\left(\cdot, x_{1}\right) \wedge \cdots \wedge d\left(\cdot, x_{i}\right)$ and $\beta^{\prime}-d\left(\cdot, x_{1}^{\prime}\right) \wedge \cdots \wedge d\left(\cdot, x_{j}^{\prime}\right), \quad(i=1, \cdots, n$; $j=1, \cdots, m)$. Secondly, for every function $f$ generated in this way, the compact set $(f \geqq-\theta$ ) belongs to $F$. Furthur, we know by assumption that $-\beta$ belongs to the complement of some exceptional set $A$ for the functions $d\left(\cdot, x_{1}\right), \cdots, d\left(\cdot, x_{n}\right)$, and we choose $\theta$ so that $-\beta-\theta$ belongs to $-A$ also; a similar relation is to hold for $-\beta^{\prime}$ and $-\beta^{\prime}-\theta$. Then the compact sets $\bar{K}_{i} \equiv\left\{x: d\left(x, x_{1}\right) \wedge \cdots \wedge d\left(x, x_{i}\right) \leqq \beta+\theta\right\}=\{x: \beta-$ $\left.d\left(x, x_{1}\right) \wedge \cdots \wedge d\left(x, x_{i}\right) \geqq-\theta\right\}$ and the compact sets $\bar{K}_{j}^{\prime} \equiv\left\{x: d\left(x, x_{1}^{\prime}\right) \wedge \cdots \wedge\right.$ $\left.d\left(x, x_{j}^{\prime}\right) \leqq \beta^{\prime}+\theta\right\}=\left\{x: \beta^{\prime}-d\left(x, x_{1}^{\prime}\right) \wedge \cdots \wedge d\left(x, x_{j}^{\prime}\right) \geqq-\theta\right\}$ will have the desired properties; namely, compact sets generated from them via $\wedge$ and $V$ belong to $F$ and are pairwise compatible. From 3.1(ii) we see that the numbers $\theta,|S-\bar{S}|$, and $\left|S^{\prime}-\bar{S}^{\prime}\right|$ can be made arbitrarily small.)

Let $L$ be a compact set outside which $g$ vanishes. Choose a number $\gamma>1$ such that $K \equiv\{x: d(x, L) \leqq \gamma)$ belongs to $F$. Since we will be concerned only with small values of $\alpha$ and $\alpha^{\prime}$, we may assume $K_{n} \subset K$ and $K_{m}^{\prime} \subset K$. Now suppose $\varepsilon>0$ is given. Let $\delta \in(0, \varepsilon)$ be so small that $d\left(x, x^{\prime}\right)<\delta$ implies $\left|g(x)-g\left(x^{\prime}\right)\right|<\varepsilon$. To estimate $\left|S-S^{\prime}\right|$, write

$$
\begin{aligned}
S= & \sum_{i=1}^{n} g\left(x_{i}\right)\left[\nu\left(K_{i}\right)-\nu\left(K_{i-1}\right)-\nu\left(K_{i} \wedge K_{m}^{\prime}\right)+\nu\left(K_{i-1} \wedge K_{m}^{\prime}\right)\right] \\
& +\sum_{i=1}^{n} g\left(x_{i}\right) \sum_{j=1}^{m}\left[\nu\left(K_{i} \wedge K_{j}^{\prime}\right)-\nu\left(K_{i} \wedge K_{j-1}^{\prime}\right)-\nu\left(K_{i-1} \wedge K_{j}^{\prime}\right)+\nu\left(K_{j-1} \wedge K_{j-1}^{\prime}\right)\right],
\end{aligned}
$$

with a similar expression for $S^{\prime}$. Then, for $\alpha, \alpha^{\prime}<\delta / 2$, we have

$$
\begin{aligned}
\left|S-S^{\prime}\right| \leqq & \sum_{i=1}^{n}\left|g\left(x_{i}\right)\left[\nu\left(K_{i}\right)-\nu\left(K_{i-1}\right)-\nu\left(K_{i} \wedge K_{m}^{\prime}\right)+\nu\left(K_{i-1} \wedge K_{m}^{\prime}\right)\right]\right| \\
& +\sum_{i=1}^{n} \sum_{\jmath=1}^{m} \mid\left(g\left(x_{i}\right)-g\left(x_{\jmath}^{\prime}\right)\right)\left[\nu\left(K_{i} \wedge K_{\jmath}^{\prime}\right)-\nu\left(K_{i} \wedge K_{j-1}^{\prime}\right)\right. \\
& \left.-\nu\left(K_{i-1} \wedge K_{\jmath}^{\prime}\right)+\nu\left(K_{i-1} \wedge K_{j-1}^{\prime}\right)\right] \mid \\
& +\sum_{\jmath=1}^{m}\left|g\left(x_{j}^{\prime}\right)\left[\nu\left(K_{j}^{\prime}\right)-\nu\left(K_{j-1}^{\prime}\right)-\nu\left(K_{j}^{\prime} \wedge K_{n}\right)+\nu\left(K_{j-1}^{\prime} \wedge K_{n}\right)\right]\right| \\
= & \sum_{i=1}^{n}\left|g\left(x_{i}\right)\left[\nu\left(K_{i} \vee K_{m}^{\prime}\right)-\nu\left(K_{i-1} \vee K_{m}^{\prime}\right)\right]\right| \\
& +\sum_{i=1}^{n} \sum_{j=1}^{m} \mid\left(g\left(x_{i}\right)-g\left(x_{j}^{\prime}\right)\right)\left[\nu\left(\left(K_{i} \wedge K_{j}^{\prime}\right) \vee\left(K_{i-1} \wedge K_{m}^{\prime}\right)\right)\right. \\
& \left.-\nu\left(\left(K_{i} \wedge K_{j-1}^{\prime}\right) \vee\left(K_{i-1} \wedge K_{m}^{\prime}\right)\right)\right] \mid
\end{aligned}
$$




$$
+\sum_{j=1}^{m}\left|g\left(x_{j}^{\prime}\right)\left[\nu\left(K_{j}^{\prime} \vee K_{n}\right)-\nu\left(K_{j-1}^{\prime} \vee K_{n}\right)\right]\right| \cdot
$$

The equality follows easily from 3.1(i). Suppose the $i$ th term in the first sum is positive. Then, in view of 3.2 , we can find $x$ in $K_{i} \vee K_{m}^{\prime}$ with $d\left(x, K_{i-1} \vee K_{m}^{\prime}\right)>0$. Obviously $d\left(x, x_{j}\right) \leqq \beta$ and $d\left(x, K_{m}^{\prime}\right)>0$. Since $K_{m}^{\prime} \supset\left(|g| \geqq \alpha^{\prime}\right)$, we must have $|g(x)| \leqq \alpha^{\prime}<\delta$ and so $\left|g\left(x_{i}\right)\right| \leqq$ $\delta+\varepsilon<2 \varepsilon$. Hence the first sum in the bound for $\left|S-S^{\prime}\right|$ is no greater than

$$
2 \varepsilon \sum_{i=1}^{n}\left|\nu\left(K_{i} \vee K_{m}^{\prime}\right)-\nu\left(K_{i-1} \vee K_{m}^{\prime}\right)\right| \leqq 2 \varepsilon B_{K},
$$

where $B_{K}$ is the constant associated to $K$ as in 3.1(iii). Similarly, the third sum is bounded by $2 \varepsilon B_{K}$. As for the second sum, if the summand indexed by $(i, j)$ is positive, then 3.2 again implies the existence of some $y$ in $\left(K_{i} \wedge K_{j}^{\prime}\right) \vee\left(K_{i-1} \wedge K_{m}\right)$ with $d\left(y,\left(K_{i} \wedge K_{j-1}^{\prime}\right) \vee\left(K_{i-1} \wedge K_{m}^{\prime}\right)\right)>0$. It follows from this that $d\left(y, x_{i}\right) \leqq \beta$ and $d\left(y, x_{j}^{\prime}\right) \leqq \beta^{\prime}$, and so $d\left(x_{i}, x_{j}^{\prime}\right) \leqq$ $\beta+\beta^{\prime}<2 \alpha<\delta$. Therefore $\left|g\left(x_{i}\right)-g\left(x_{j}^{\prime}\right)\right| \leqq \varepsilon$. Hence the second sum is bounded by

$$
\varepsilon \sum_{i=1}^{n} \sum_{j=1}^{m}\left|\nu\left(\left(K_{i} \wedge K_{j}^{\prime}\right) \vee\left(K_{i-1} \wedge K_{m}^{\prime}\right)\right)-\nu\left(\left(K_{i} \wedge K_{j-1}^{\prime}\right) \vee\left(K_{i-1} \wedge K_{m}^{\prime}\right)\right)\right| \leqq \varepsilon B_{K} .
$$

(Here we used the fact that for every $i$,

$$
\begin{aligned}
& K_{i} \wedge K_{m}^{\prime} \supset \cdots \supset\left(K_{i} \wedge K_{j}^{\prime}\right) \vee\left(K_{i-1} \wedge K_{m}^{\prime}\right) \supset\left(K_{i} \wedge K_{j-1}^{\prime}\right) \\
& \left.\quad \vee\left(K_{i-1} \wedge K_{m}^{\prime}\right) \supset \cdots \supset\left(K_{i-1} \wedge K_{m}^{\prime}\right) .\right)
\end{aligned}
$$

Summing up, we have $\left|S-S^{\prime}\right| \leqq 5 \varepsilon B_{K}$. Since $\varepsilon>0$ was arbitrary, the desired convergence follows.

Proposition 3.4. If we define a function $\bar{\nu}$ on $C(X)$ by $\bar{\nu}(g) \equiv$ $\int g d \bar{\nu} \equiv \lim _{\alpha \rightarrow 0} S$, then $\bar{\nu}$ is an integral.

Proof. The linearity of $\bar{\nu}$ is obvious from the definition of $S$. Suppose now $f \in C(X)$ is supported by the compact set $L$. Let $K$ be associated to $L$ as in the proof of 3.3. Let $g$ be any test function such that $|g| \leqq|f|$. Then $g$ is also supported by $L$, and by the definition of $S$ we have $|S| \leqq\|g\| B_{K}$ as soon as $\alpha$ is so small that $K_{n} \subset K$. Thus $\mid \int g d \bar{\nu} \leqq\|g\| B_{K} \leqq\|f\| B_{K}$. Therefore 2.1 is satisfied.

We now prove the following Riesz Representation Theorem for a signed integral on $(X, d)$. This theorem also shows that the family of measure spaces as defined in 3.1 is not vacuous.

Proposition 3.5. Let $\mu$ be an integral on $(X, d)$. Let $F$ be the 
family of compact subsets of $X$ which are strongly measurable with respect to $\mu$, and let $\nu(K)$ be the strong measure of $K$ for each $K$ in $F$. Then $(X, d, F, \nu)$ is a measure space, and for each $g$ in $C(X)$ we have $\int g d \mu=\int g d \bar{\nu}$, where $\bar{\nu}$ is the integral defined in 3.4 .

Proof. That $(X, d, F, \nu)$ is a measure space follows from 2.10, 2.11, 2.13, 2.14, and 2.15. To show that $\mu$ and $\bar{\nu}$ coincide on $C(X)$ consider any continuous function $g$ which vanishes outside some compact set $L$. Consider a sum $S=\sum_{i=1}^{n} g\left(x_{i}\right)\left(\nu\left(K_{i}\right)-\nu\left(K_{i-1}\right)\right)$ where $x_{1}, \cdots, x_{n}$ form a $\beta$-net for $(|g| \geqq \alpha)$ with $\beta<\alpha$. For each $i$ let $\mathrm{g}_{m}^{i}$ be a sequence of test functions belonging to $K_{i}$ such that $\int g_{m}^{i} d \mu$ converges to $\nu\left(K_{i}\right)$ as $m$ approaches infinity. We may assume that $g_{m}^{i-1} \leqq g_{m}^{i} \leqq f$ where $f$ is a nonnegative test function with $f(x)=1$ whenever $d(x, L) \leqq 1$. Suppose $\varepsilon>0$ is given. Let $\alpha<\varepsilon$ be so small, and $m$ so large, that $d\left(x, x^{\prime}\right) \leqq m^{-1}+\alpha$ implies $\left|g(x)-g\left(x^{\prime}\right)\right|<\varepsilon$. Consider a point $x$ in $K_{n}$. We have $\sum_{i=1}^{n}\left(g_{m}^{i}(x)-g_{m}^{i-1}(x)\right)=g_{m}^{n}(x)=1$. $\left(\right.$ Here $\left.g_{m}^{0} \equiv 0\right)$. Therefore

$$
g(x)-\sum_{i=1}^{n} g\left(x_{i}\right)\left(g_{m}^{i}(x)-g_{m}^{i-1}(x)\right)=\sum_{i=1}^{n}\left(g(x)-g\left(x_{i}\right)\right)\left(g_{m}^{i}(x)-g_{m}^{i-1}(x)\right) .
$$

If the $i$ th term is unequal to 0 , then we must have $d\left(x, x_{i}\right) \leqq m^{-1}+\beta$ and so $\left|g(x)-g\left(x_{i}\right)\right| \leqq \varepsilon$. Therefore the above sum is bounded in absolute value by $\varepsilon \sum_{i=1}^{n}\left(g_{m}^{i}(x)-g_{m}^{i-1}(x)\right)=\varepsilon$. Next consider a point $x$ with $d\left(x, K_{n}\right)>0$. Then $|g(x)| \leqq \alpha<\varepsilon$. If the $i$ th term in $\sum_{i=1}^{n} g\left(x_{i}\right)\left(g_{m}^{i}(x)-\right.$ $\left.g_{m}^{i-1}(x)\right)$ is unequal to 0 , then we must have $d\left(x, x_{i}\right)<m^{-1}+\beta$ and so $\left|g\left(x_{i}\right)\right| \leqq|g(x)|+\varepsilon<2 \varepsilon$. Hence $\left|g(x)-\sum_{i=1}^{n} g\left(x_{i}\right)\left(g_{m}^{i}(x)-g_{m}^{i-1}(x)\right)\right|<3 \varepsilon$.

In view of the continuity of $g$ and the functions $g_{m}^{i}$, we conclude that the function $g-\sum_{i=1}^{n} g\left(x_{i}\right)\left(g_{m}^{i}-g_{m}^{i-1}\right)$ is always bounded in absolute value by $3 \varepsilon$. This function is therefore bounded in absolute value by the function $3 \varepsilon f$. Its integral $\int g d \mu-\int \sum_{i=1}^{n} g\left(x_{i}\right)\left(g_{m}^{i}-g_{m}^{i-1}\right) d \mu$ must then be bounded in absolute value by $3 \varepsilon M_{f}$. $\quad\left(M_{f}\right.$ is the constant associated to $f$ in 2.1). Letting $m \rightarrow \infty$, we have $\left|\int g d \mu-S\right| \leqq 3 \varepsilon M_{f}$. Then, letting $\alpha \rightarrow 0$, we have $\left|\int g d \mu-\int g d \bar{\nu}\right| \leqq 3 \varepsilon M_{f}$. But $\varepsilon$ was arbitrary. The integrals $\mu$ and $\bar{\nu}$ are equal.

The author wishes to thank Professor E. A. Bishop for his guidance, advice, and encouragement during the time when this research was being done.

\section{REFERENCE}

E. A. Bishop, Foundations of Constructive Analysis, McGraw Hill, 1967. 
Received June 29, 1970. Part of a dissertation submitted in partial satisfaction of the requirements for the degree Doctor of Philosophy in Mathematics at the University of California, San Diego. This work was partially supported by N.S.F. Grant G.P. 5094. UNIVERSITY OF WASHINGTON 



\section{PACIFIC JOURNAL OF MATHEMATICS}

\section{EDITORS}

H. SAMELSON

Stanford University

Stanford, California 94305

C. R. Hовву

University of Washington

Seattle, Washington 98105
J. DugundJI

Department of Mathematics

University of Southern California

Los Angeles, California 90007

RICHARD ARENS

University of California

Los Angeles, California 90024

\section{ASSOCIATE EDITORS}
E. F. BECKENBACH
B. H. NeumanN
F. WOLF
K. YOSHIDA

\section{SUPPORTING INSTITUTIONS}

UNIVERSITY OF BRITISH COLUMBIA

CALIFORNIA INSTITUTE OF TECHNOLOGY

UNIVERSITY OF CALIFORNIA

MONTANA STATE UNIVERSITY

UNIVERSITY OF NEVADA

NEW MEXICO STATE UNIVERSITY

OREGON STATE UNIVERSITY

UNIVERSITY OF OREGON

OSAKA UNIVERSITY
UNIVERSITY OF SOUTHERN CALIFORNIA

STANFORD UNIVERSITY

UNIVERSITY OF TOKYO

UNIVERSITY OF UTAH

WASHINGTON STATE UNIVERSITY

UNIVERSITY OF WASHINGTON

$\stackrel{*}{*} \stackrel{*}{*} \stackrel{*}{*}{ }^{*}$ AMERICAN MATHEMATICAL SOCIETY

NAVAL WEAPONS CENTER 


\section{Pacific Journal of Mathematics}

\section{Vol. 41, No. $1 \quad$ November, 1972}

Anatole Beck and Peter Warren, Weak orthogonality.................

Jonnie Bee Bednar and Howard E. Lacey, Concerning Banach spaces whose duals are abstract $L$-spaces.............................

Louis Harvey Blake, Canonical extensions of measures and the extension of regularity of conditional probabilities..........................

R. A. Brooks, Conditional expectations associated with stochastic processes ..........................................

Theodore Allen Burton and Ronald Calvin Grimmer, On the asymptotic behavior of solutions of $x^{\prime \prime}+a(t) f(x)=e(t) \ldots \ldots \ldots \ldots \ldots \ldots$

Stephen LaVern Campbell, Operator-valued inner functions analytic on the closed disc ............................................

Yuen-Kwok Chan, A constructive study of measure theory...

Alexander Munro Davie and Bernt Karsten Oksendal, Peak interpolation sets for some algebras of analytic functions ...................

H. P. Dikshit, Absolute total-effective $\left(N, p_{n}\right)(c, 1)$ method ...............

Robert E. Edwards, Edwin Hewitt and Kenneth Allen Ross, Lacunarity for

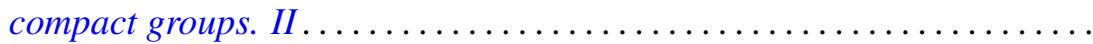

James Daniel Halpern, On a question of Tarski and a maximal theorem of Kurepa

Gerald L. Itzkowitz, A characterization of a class of uniform spaces that admit an invariant integral

Mo Tak Kiang, Semigroups with diminishing orbital diameters ..

Glenn Richard Luecke, A class of operators on Hilbert space ...

R. James Milgram, Group representations and the Adams spectral sequence. . .

G. S. Monk, On the endomorphism ring of an abelian p-group, and of a large subgroup...

Yasutoshi Nomura, Homology of a group extension ...

R. Michael Range, Approximation to bounded holomorphic functions on strictly pseudoconvex domains...

Norman R. Reilly, Inverse semigroups of partial transformations and $\theta$-classes.

Chris Rorres, Strong concentration of the spectra of self-adjoint operators

Saharon Shelah, A combinatorial problem; stability and order for models and theories in infinitary languages.

George Gustave Weill, Vector space decompositions and the abstract

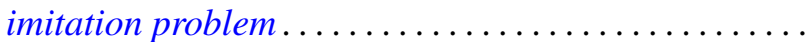

